\title{
Inhibition of microRNA-16 protects mesenchymal stem cells against apoptosis
}

\author{
JIANG RUI ${ }^{1,2}$, SHAOHONG FANG ${ }^{2,3}$, YONGCHEN WANG ${ }^{1}$, BO LV ${ }^{2,3}$, BO YU ${ }^{2,3}$ and SHUFENG $\mathrm{LI}^{2,3}$ \\ ${ }^{1}$ General Practice Department; ${ }^{2}$ Key Laboratory of The Education Ministry for Myocardial Ischemia Mechanism \\ and Treatment; ${ }^{3}$ Department of Cardiology, The Second Affiliated Hospital of Harbin Medical University, \\ Harbin, Heilongjiang 150086, P.R. China
}

Received November 5, 2017; Accepted April 13, 2018

DOI: $10.3892 / \mathrm{mmr} .2018 .9012$

\begin{abstract}
Bone marrow-derived mesenchymal stem cells (BM-MSCs) have been used in experimental research and clinical trials for heart function restoration and cardiomyocyte regeneration. However, due to a hostile microenvironment created by ischemia, hypoxia and pro-inflammatory factors, the survival rate of implanted BM-MSCs remains low. Therefore, strategies that can promote BM-MSC survival and prevent apoptosis are required. Previous studies have reported that microRNA-16 (miR-16) can inhibit cell proliferation by targeting several proteins and signal pathway, not only by inducing apoptosis. In the present study, it was investigated whether inhibition of miR-16 reduced BM-MSC apoptosis in a model of ischemia. Flow cytometry analysis revealed that BM-MSCs underwent apoptosis in response to hypoxia/serum deprivation (SD). Additionally, in hypoxic/SD conditions, miR-16 expression increased and B-cell lymphoma (Bcl)-2 protein expression decreased in BM-MSCs. miR-16 did not affect Bcl-2 mRNA expression but downregulated Bcl-2 protein expression. miR-16 inhibitor transfection significantly increased $\mathrm{Bcl}-2$ protein expression and the percentage of apoptotic BM-MSCs was reduced. The pro-apoptotic effects of miR-16 were partially elevated by knocking down of Bcl-2. Furthermore, it was demonstrated that miR-16 exerted its pro-apoptotic effects by activating the mitochondrial pathway of apoptosis via apoptotic protease activating factor-1/caspase-9/poly (ADP ribose) polymerase. Taken together, the results indicated that miR-16 downregulated $\mathrm{Bcl}-2$ expression and promoted BM-MSC apoptosis, indicating that
\end{abstract}

Correspondence to: Dr Shufeng Li, Key Laboratory of The Education Ministry for Myocardial Ischemia Mechanism and Treatment, The Second Affiliated Hospital of Harbin Medical University, 148 Baojian Road, Harbin, Heilongjiang 150086, P.R. China

E-mail: lishufengep@163.com

Key words: bone marrow-derived mesenchymal stem cells, microRNA-16, B-cell lymphoma 2, apoptosis, hypoxia and serum deprivation, proliferation therapies targeting miR-16 may improve the effectiveness of BM-MSC transplantation therapy.

\section{Introduction}

Bone marrow-derived mesenchymal stem cells (BM-MSCs) are capable of self-renewal and multilineage differentiation into various cell types (1-3), including endothelial cells, neural cells, smooth muscle cells, skeletal myoblasts, and cardiac myocytes $(4,5)$. Considerable experimental and clinical evidence has demonstrated that infracted myocardium regeneration with BM-MSCs is safe and feasible (2). Transplantation of BM-MSCs leads to the improved neovascularization of ischemic myocardium and myocardial fibrosis inhibition, as well as an increase in prosurvival growth factor secretion, including vascular endothelial growth factor, hepatocyte growth factor, and insulin-like growth factor (5-8). Despite these advantages, the poor survival rate of BM-MSCs within the first few days following engraftment in the infarcted heart results in only marginal functional improvement $(9,10)$. Therefore, it is necessary to protect the cells against the hostile microenvironment created by ischemia, hypoxia, the inflammatory response and pro-apoptotic factors, in order to improve the efficacy of BM-MSC transplantation therapy.

microRNAs (miRNAs/miRs) are endogenous RNAs $\sim 22$ nucleotides in length that negatively regulate gene expression by targeting mRNAs for cleavage or translational repression, which occurs primarily through base pairing to the 3' untranslated region (UTR) of target mRNAs $(11,12)$. Previous studies have reported that miRNA-regulated translational repression appears to occur on the post-transcriptional level via inhibition of protein translation from mRNAs or by promotion of mRNA degradation $(13,14)$. Furthermore, bioinformatics analyses have predicted that each miRNA regulates hundreds of targets, suggesting that miRNAs may have a central role in an increasing number of biological processes, including cell proliferation, migration, differentiation and apoptosis $(11,14,15)$. Additionally, it has been demonstrated that miRNAs are involved in the self-renewal and differentiation of MSCs (16).

Among the known miRNAs, miR-16 has been reported to regulate apoptosis and the cell cycle by targeting cyclin D3 (CCND3), cyclin E1 (CCNE1), CDK6 and B-cell 
lymphoma (Bcl)-2 in tumor cells $(15,17-20)$. Bcl-2, originally found to be overexpressed in B-cell lymphoma (21), is a critical inhibitor in the apoptotic pathway (22). Previous studies have demonstrated that Bcl-2 is an integral membrane protein that resides on the outer membrane of mitochondria. It prevents the initiation of the cellular apoptotic program by blocking cytochrome $\mathrm{c}$ release from mitochondria in response to a variety of stimuli $(23,24)$. Cytochrome $\mathrm{c}$ is a key mediator of apoptosis that may bind to apoptotic protease activating factor-1 (APAF-1), which subsequently activates caspase-9 (25).

Accumulating evidence suggests that miR-16 targets Bcl-2 at the posttranscriptional level (18-20). Additionally, miR-16 downregulation in various cell types coincides with Bcl-2 upregulation $(19,20)$. However, if miR-16 functions as a regulator of apoptosis in stem cells remains unknown. In the current study, it was hypothesized that miR-16 inhibition may protect BM-MSCs from apoptosis. It was investigated whether miR-16, which is among the upregulated miRNAs in a hypoxic/serum deprived (SD) environment, regulated the apoptosis of BM-MSCs by targeting Bcl-2. The data indicated that inhibition of miR-16 resulted in increased $\mathrm{Bcl}-2$ protein expression and a reduced rate of apoptosis. Additionally, it was demonstrated that miR-16 regulated mitochondrial apoptosis through APAF-1/caspase-9/poly (ADP ribose) polymerase (PARP) pathway.

\section{Materials and methods}

Animals and ethics statement. Male Sprague-Dawley rats weighing 60-80 g (8-10 weeks old) were housed under standardized conditions at $22^{\circ} \mathrm{C}$ in a 12 -h light/dark cycle and fed a laboratory diet with water ad libitum. They were obtained from the Laboratory Animal Science Department of the Second Affiliated Hospital of Harbin Medical University (Harbin, China). All experimental animal procedures were approved by the Ethical Committee on Animal Care and Use of Harbin Medical University (Harbin, China).

BM-MSC culture and treatment. BM-MSCs were cultured using the whole bone marrow adherent method, as previously reported (4). Briefly, total bone marrow was harvested from the tibia and femur of rats. BM-MSCs were placed in $25 \mathrm{~cm}^{2}$ culture flasks at a density of $10^{6}$ cells $/ \mathrm{ml}$ in Dulbecco's modified Eagle's medium/nutrient mixture F12 (DMEM/F12; Thermo Fisher Scientific, Inc., Waltham, MA, USA) supplemented with $15 \%$ fetal bovine serum (FBS; Thermo Fisher Scientific, Inc.) and $1 \%$ penicillin/streptomycin. Cells were incubated at $37^{\circ} \mathrm{C}$ in a humidified tissue culture incubator with $5 \% \mathrm{CO}_{2}$. After 3 days of incubation, the medium was changed and then replaced every 2 days thereafter. Passage 3 BM-MSCs were used in all experiments.

Hypoxia and SD treatment. Apoptosis was induced by hypoxia and SD in vitro. The method was designed to mimic the in vivo conditions of ischemia in the myocardium and was performed as previously described (26). In brief, BM-MSCs were washed with serum-free DMEM/F12 and incubated in a $5 \% \mathrm{CO}_{2} / 95 \% \mathrm{~N}_{2}$ incubator (Controlled Atmosphere Chamber, PLAS-Labs, Inc., Lansing, MI, USA) for 3-24 h. BM-MSCs incubated in a $5 \% \mathrm{CO}_{2} / 95 \% \mathrm{O}_{2}$ incubator were used as the normoxic control and were cultured in DMEM/F12 supplemented with $15 \%$ FBS and $1 \%$ penicillin/streptomycin.

Cell transfection. Small interfering RNAs (siRNAs) are small double-stranded RNAs that target mRNA to silence its expression. A Bcl-2 siRNA duplex was synthesized by Thermo Fisher Scientific, Inc. (sense, 5'-GCUGCACCUGAC GCCCUUCTT-3' and antisense, 3'-TTCGACGUGGACUGC GGGAAG-5'). Cells were transfected using X-tremeGENE ${ }^{\mathrm{TM}}$ siRNA Transfection Reagent (Sigma-Aldrich; Merck KGaA, Darmstadt, Germany) as previously described (27). Cells were seeded in a 6 -well plate $\left(2 \times 10^{5}\right.$ cells/well) and incubated at $37^{\circ} \mathrm{C}$ for $24 \mathrm{~h}$ and subsequently transfected with miR-16 mimics, miR-16 mimic inhibitor, scrambled miRNA, or Bcl-2 siRNA (100 nM). siRNA (GCUGCACCUGACGCCCUU CTT; TTCGACGUGGACUGCGGGAAG); Scramble (UUC UCCGAACGUGUCUCG; TTAAGAGGCUUGCACAGU GCA; all from Invitrogen; Thermo Fisher Scientific, Inc.) and incubated in $2 \mathrm{ml} \mathrm{FBS}$-free Opti-MEM (Invitrogen; Thermo Fisher Scientific, Inc.) for 6-8 h. All cells were subjected to hypoxia and SD treatment prior to transfection. Following this, the medium was replaced with fresh complete medium and the cells were incubated for an additional $24 \mathrm{~h}$. Transfected cells were subjected to analysis $72 \mathrm{~h}$ post-transfection.

Cell viability and proliferation assays. BM-MSC viability and proliferation was determined using an MTT assay (Sigma-Aldrich; Merck KGaA) and an EdU incorporation assay (Guangzhou RiboBio Co., Ltd., Guangzhou, China), respectively, according to the manufacturers' protocols. For the MTT assay, cells were seeded into a 96-well plate (3,000 cells/well), and viability was detected with the addition of $20 \mu \mathrm{l} \mathrm{MTT}(5 \mathrm{mg} / \mathrm{ml})$, dissolved in DMSO, to the culture medium. The absorbance of each well was quantified at $490 \mathrm{~nm}$ using the Infinite M200 PRO plate reader (Tecan, Morrisville, $\mathrm{NC}$, USA). All data were calculated from triplicate samples and are presented as the mean \pm standard deviation.

For the EdU incorporation assay, BM-MSCs were cultured in 96-well plates at a density of $4 \times 10^{3}$ cells/well for $24 \mathrm{~h}$ at $37^{\circ} \mathrm{C}$. Following this, $50 \mu \mathrm{M}$ EdU was added to each well and cells were cultured for additional $2 \mathrm{~h}$ at $37^{\circ} \mathrm{C}$. Cells were fixed with $4 \%$ formaldehyde for $15 \mathrm{~min}$ at room temperature and subsequently treated with $0.5 \%$ Triton X-100 for 20 min for permeabilization. Following three washes with PBS, $100 \mu 1$ $1 \mathrm{X}$ Apollo reaction cocktail was added to each well and the cells were incubated for $30 \mathrm{~min}$ at room temperature prior to staining with $100 \mu \mathrm{l}$ Hoechst $33342(10 \mu \mathrm{g} / \mathrm{ml})$ at room temperature $\left(24^{\circ} \mathrm{C}\right)$ for $30 \mathrm{~min}$ and visualization under a fluorescent microscope (magnification, x 100; Leica Microsystems $\mathrm{GmbH}$, Wetzlar, Germany). The positive staining rate (\%) was counted as positive cells (green cells)/overall cells (blue cells). DAPI $(50 \mu \mathrm{g} / \mathrm{ml})$ stain was conducted in $37^{\circ} \mathrm{C}$ for $2 \mathrm{~h}$. Cells were counted from 6 random fields in triplicate wells for each condition and expressed as percentage of total number of cells in the field. All experiments were performed in triplicate and three independent repeated experiments were performed.

RNA extraction and reverse transcription-quantitative polymerase chain reaction ( $R T-q P C R)$. Total RNA was extracted from the BM-MSCs with TRIzol reagent (Invitrogen; Thermo 

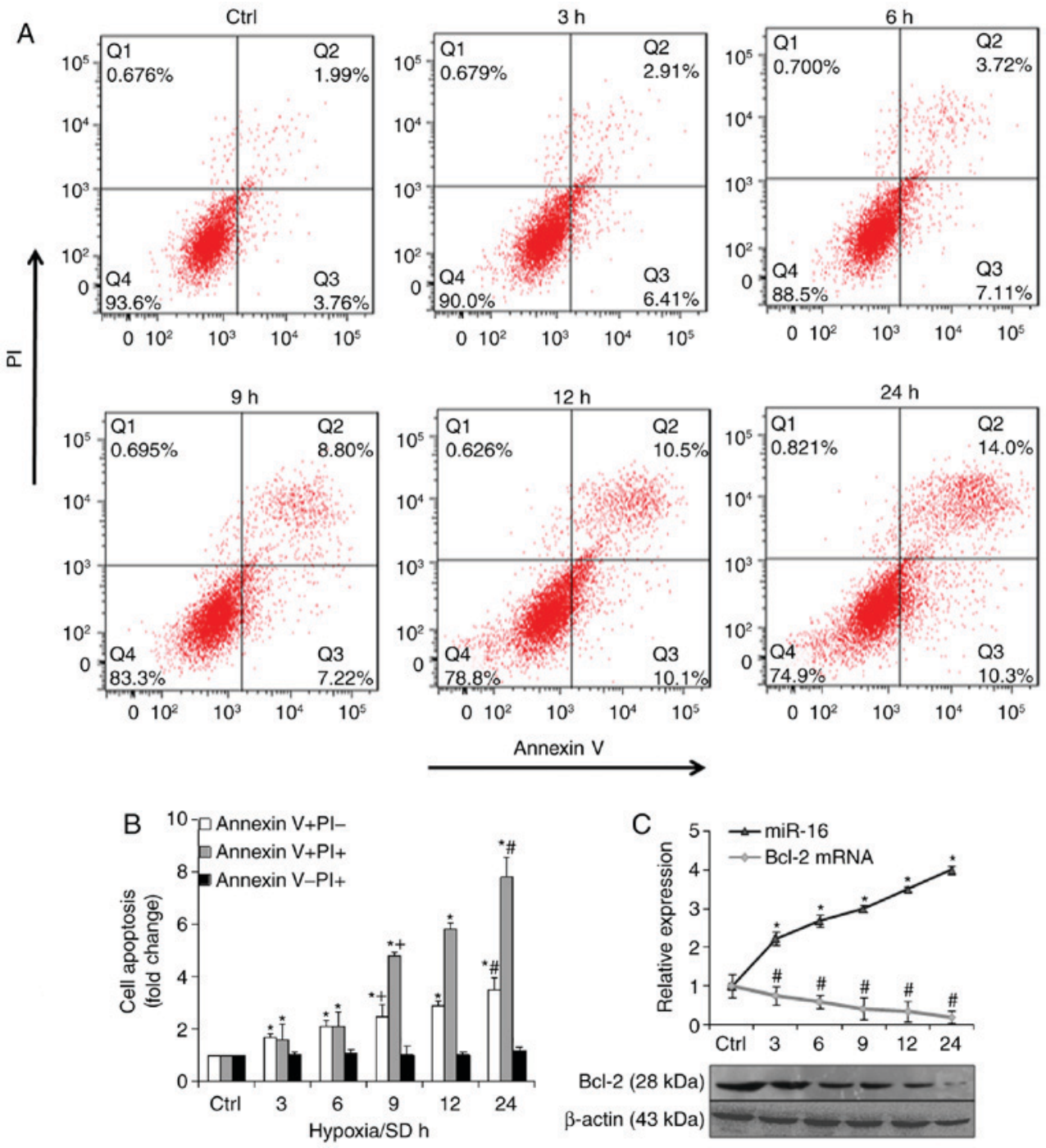

$\mathrm{Bcl}-2(28 \mathrm{kDa})$

$\beta$-actin $(43 \mathrm{kDa})$

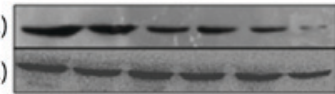

Figure 1. miR-16 expression in hypoxia/SD-induced apoptosis. (A) Cells were stained with Annexin V and PI and analyzed by flow cytometry. The percentage of cells in each group within the gated areas is indicated; the upper right panel represents cells undergoing late apoptosis, and the lower right panel represents cells undergoing early apoptosis. (B) Fold change in apoptosis. Data are expressed as the mean \pm standard deviation of three independent experiments. ${ }^{*} \mathrm{P}<0.05$ vs. ctrl, ${ }^{+} \mathrm{P}<0.05$ vs. the respective group at $3 \mathrm{~h},{ }^{\#} \mathrm{P}<0.05$ vs. the respective group at $9 \mathrm{~h}$. (C) Quantitative analysis of miR-16 and Bcl-2 mRNA and protein expression by reverse transcription-quantitative polymerase chain reaction and western blot analysis. Fold change compared with the expression levels in corresponding control cells are presented. Each data point represents the mean \pm standard deviation of three independent experiments. "P<0.05 and ${ }^{\#} \mathrm{P}<0.05$ vs. Ctrl. SD, serum deprivation; PI, propidium iodide; Bcl-2, B-cell lymphoma 2; miR-16, microRNA-16; Ctrl, control.

Fisher Scientific, Inc.) and reverse transcribed into cDNA with the miRcute miRNA first-strand cDNA synthesis kit (Tiangen Biotech Co., Ltd., Beijing, China) according to the manufacturer's instructions. PCR (Initial Denaturation, $98^{\circ} \mathrm{C}$, $30 \mathrm{sec}, 25-35$ cycles at $98^{\circ} \mathrm{C}, 5-10 \mathrm{sec} ; 45-72^{\circ} \mathrm{C}, 10-30 \mathrm{sec}$; $72^{\circ} \mathrm{C}, 15-30 \mathrm{sec}$ per kb. Final Extension, $72^{\circ} \mathrm{C}, 5-10 \mathrm{~min}$. Hold, $4-10^{\circ} \mathrm{C}$.) was performed to analyze the level of miR-16 using the miRcute miRNA qPCR Detection kit (SYBR Green; Tiangen Biotech Co., Ltd.). All primers for miR-16 and U6 for the TaqMan miRNA assays were purchased from Thermo Fisher Scientific, Inc. The miR-16 primer sequences were as follows: forward, 5'-GCTTCGGCAGCACATATACTAAAAT-3' and reverse, 5'-CGCTTCACGAATTTGCGTGTCAT-3'. The relative expression level of miR-16 was normalized to that of the internal control U6 using the $2^{-\Delta \Delta \mathrm{Cq}}$ cycle threshold method (28).

Protein extraction and western blot analysis. Cells were washed twice with ice-cold PBS $72 \mathrm{~h}$ after transfection and harvested for further analysis. Total protein was obtained with RIPA protein extraction buffer (Thermo Fisher) and the concentration was analyzed using the bicinchoninic acid protein assay and $50 \mu \mathrm{g}$ total protein per lane, extracted with ice-cold PBS was resolved via $12 \%$ SDS-PAGE and subsequently transferred onto polyvinylidene difluoride membranes. Non-specific binding was inhibited by incubating the membranes with $5 \%$ skim milk and Tris buffered saline with $0.5 \%$ Tween-20 (TBST) at $4^{\circ} \mathrm{C}$ for $12 \mathrm{~h}$. Membranes were incubated with the following primary antibodies overnight at $4^{\circ} \mathrm{C}$ : Bcl-2, caspase-3 (1:1,000; cat no. ab49822), caspase-9 (1:1,000; cat. no. ab61789; both Abcam, Cambridge, UK), APAF-1 (1:1,000), cleaved PARP (1:1,000; Cell Signaling Technology; Danvers, MA, USA) and $\beta$-actin $(1: 1,000$; OriGene Technologies, Inc., Beijing, China). The membranes were washed with TBS-T and subsequently incubated with horseradish peroxidase-conjugated Affinipure goat anti-rabbit IgG and anti-mouse IgG secondary antibodies 
A

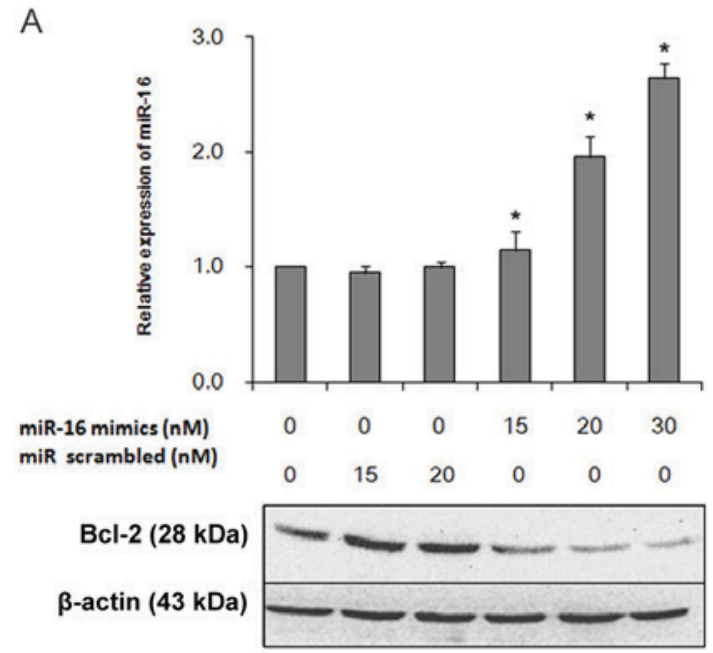

C

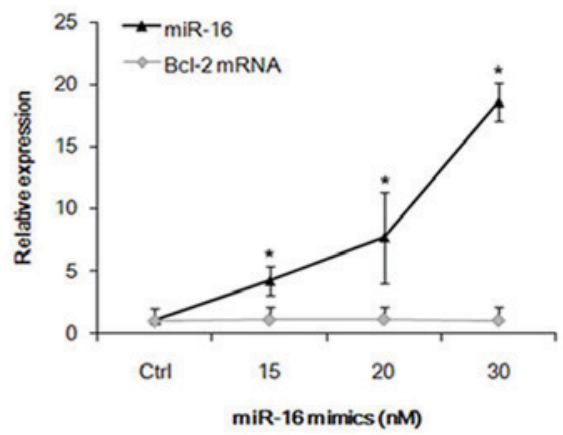

B
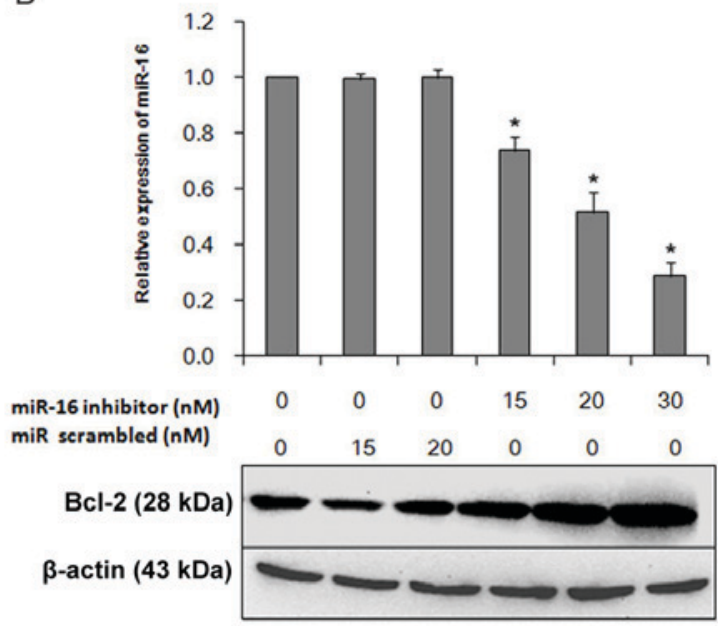

D

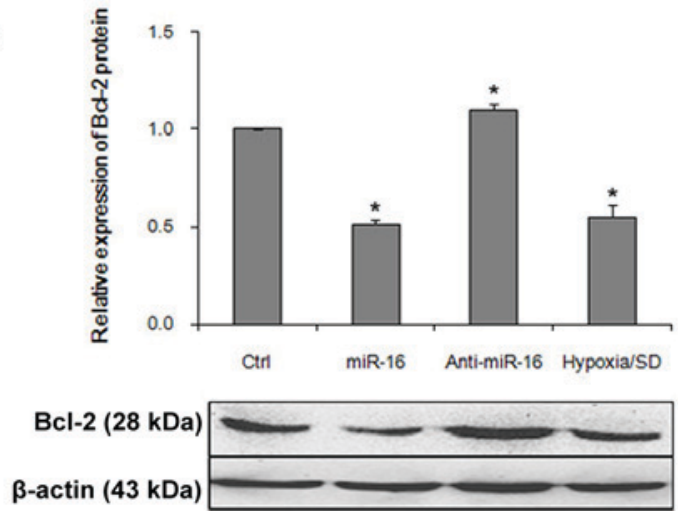

Figure 2. Bcl-2 was confirmed a target of miR-16 and its expression was inversely proportional to miR-16 expression. (A) BM-MSCs were transfected with a miR-16 mimic or (B) miR-16 inhibitor (15-30 nM). Protein expression was calculated by densitometric analysis and experiments were performed in triplicate. ${ }^{*} \mathrm{P}<0.05$ vs. Ctrl. (C) Reverse transcription-quantitative polymerase chain reaction analysis of miR-16 and Bcl-2 mRNA expression in BM-MSCs following transfection with miRNA mimics $(0,15,20$ and $30 \mathrm{nM})$ for $24 \mathrm{~h} .{ }^{*} \mathrm{P}<0.05$ vs. Ctrl group. (D) Protein expression of Bcl-2 following transfection with either a miR-16 mimic or inhibitor, or under hypoxic/SD conditions. Densitometric analysis was performed to calculate relative protein levels. All data are presented as the mean \pm standard deviation of three independent experiments. "P $<0.05$ vs. Ctrl group. B-cell lymphoma 2; miR-16, microRNA-16; BM-MSC, bone marrow-derived mesenchymal stem cell; SD, serum deprivation; Ctrl, control.

(1:5,000; TA140003; OriGene Technologies, Inc.) for $1 \mathrm{~h}$ at $37^{\circ} \mathrm{C}$. Specific complexes were visualized on an X-ray film via enhanced chemiluminescence (ECL) detection with BeyoECL Plus (Beyotime Institute of Biotechnology, Beijing, China) according to the manufacturer's protocol. Densitometric analysis was performed using a GS-710 Imaging Densitometer (Bio-Rad Laboratories, Inc., Hercules, CA, USA) and Image Lab ${ }^{\text {TM }}$ Software (170-9690) to measure protein levels normalized to that of internal control $\beta$-actin. All data were obtained in triplicate, independent experiments.

Flow cytometric evaluation of apoptosis. Cell apoptosis was examined using an Annexin V-fluorescein isothiocyanate (FITC) Apoptosis Detection kit (BD Biosciences, Franklin Lakes, NJ, USA). Briefly, cells were incubated with $5 \mu$ l Annexin V-FITC solution in the dark at room temperature for $15 \mathrm{~min}$, followed by staining with $5 \mu \mathrm{l}$ propidium iodide (PI) at room temperature for $5 \mathrm{~min}$, according to the manufacturer's protocol. Stained cells were distinguished as viable (Annexin $\left.\mathrm{V} / \mathrm{PI}^{-}\right)$, dead (Annexin $\mathrm{V} / \mathrm{PI}^{+}$), early apoptotic (Annexin $\left.\mathrm{V}^{+} / \mathrm{PI}\right)$, or late apoptotic $\left(\right.$ Annexin $\left.\mathrm{V}^{+} / \mathrm{PI}^{+}\right)$using a
FACSCalibur flow cytometer. Data were analyzed with the BD FACSCanto II equipped with BD FACSDiva software version 8.0 (BD Biosciences). Experiments were done in triplicate.

Mitochondrial membrane potential (MMP) assay. The MMP was analyzed using the JC-1 MMP Assay kit (Beyotime Institute of Biotechnology) according to the manufacturer's protocol (29). Cells were collected, resuspended in culture medium (DMEM/F12 supplemented with $15 \%$ FBS and $1 \%$ penicillin/streptomycin) to a concentration of $10^{6}$ cells $/ \mathrm{ml}$ and incubated with staining dye at $37^{\circ} \mathrm{C}$ for $15 \mathrm{~min}$. Following this, cells were washed twice with cold JC-1 staining buffer and were observed under a fluorescence microscope (magnification, x100; DMI4000B; Leica, Wetzlar, Germany).

Statistical analysis. All data are expressed as the mean \pm standard deviation. Differences between the mean values were calculated with one way analysis of variance with Fisher's LSD used for the post-hoc multiple comparisons test. Statistical analyses were performed using SPSS software 
A

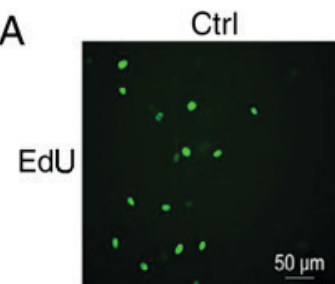

$50 \mu \mathrm{m}$
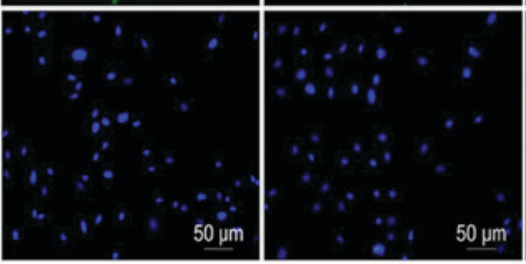

$50 \mu \mathrm{m}$
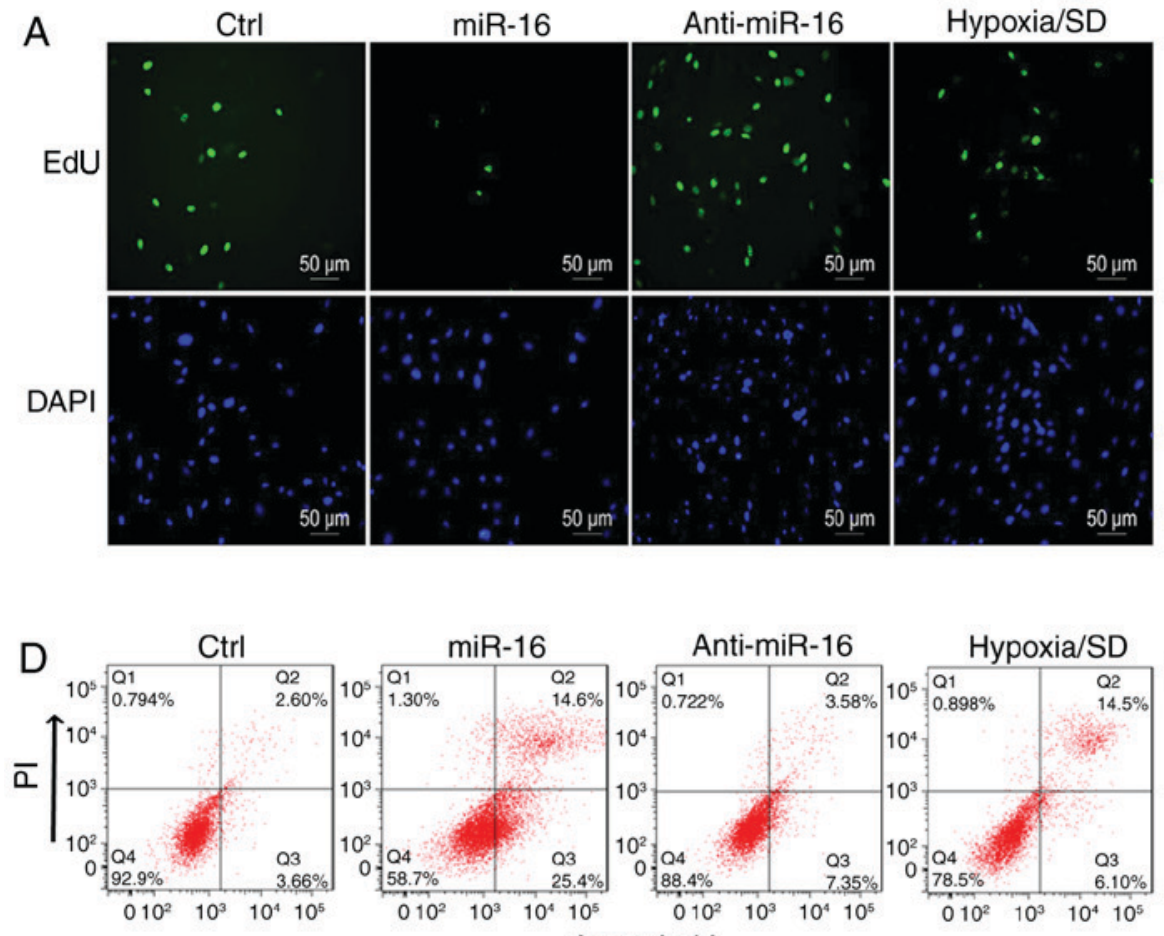

Annexin V
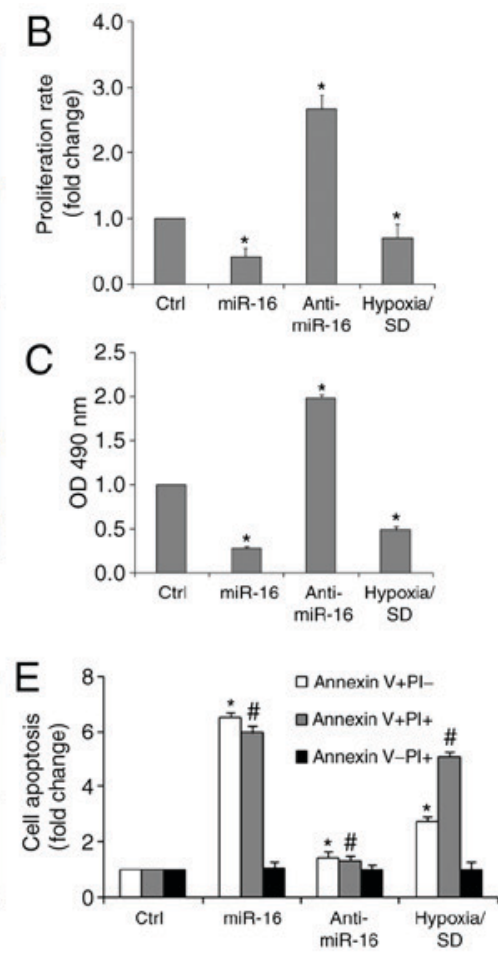

Figure 3. Effects of miR-16 on proliferation and hypoxia/SD-induced apoptosis. (A) Representative images from the EdU incorporation assay. Magnification, x100. (B) The proliferation rate of BM-MSCs transfected with miR-16 inhibitor was significantly higher compared with the other experimental groups. ${ }^{*} \mathrm{P}<0.05$ vs. Ctrl group, ${ }^{\#} \mathrm{P}<0.05$ vs. the anti-miR-16 group. (C) BM-MSC viability following miR-16 mimic or inhibitor transfection. (D) Flow cytometry analysis of apoptotic cells following Annexin V/PI staining. (E) Fold change in cell apoptosis. Data are presented as the mean \pm standard deviation of three independent experiments. ${ }^{*} \mathrm{P}<0.05$ and ${ }^{\#} \mathrm{P}<0.05$ vs. the respective $\mathrm{Ctrl}$ group. miR-16, microRNA-16; SD, serum deprivation; BM-MSC, bone marrow-derived mesenchymal stem cell; PI, propidium iodide; Ctrl, control.

(version 22.0; IBM Corp., Armonk, NY, USA). P<0.05 was considered to indicate a statistically significant difference.

\section{Results}

miR-16 expression after hypoxia/SD. To determine if miR-16 was involved in hypoxia/SD-induced apoptosis, BM-MSCs were exposed to hypoxic/SD conditions for different periods of time (3-24 h). Apoptosis was detected by Annexin V-FITC, which binds to phosphatidylserine, a phospholipid that is redistributed from the inner to the outer leaflet of the cell membrane in early apoptosis. Following membrane integrity loss, PI may also enter the cell and intercalate into DNA (30). The results revealed that hypoxia/SD induced BM-MSCs to undergo apoptosis (Fig. 1A). Notably, apoptosis (Annexin $\mathrm{V}^{+} / \mathrm{PI}$ ) significantly increased with time (Fig. 1B). RT-qPCR and western blot analyses were used to evaluate expression levels of miR-16 and Bcl-2. The results indicated that in hypoxia/SD conditions, miR-16 expression was markedly upregulated and Bcl-2 mRNA and protein expression was markedly downregulated, in a time-dependent manner (Fig. 1C).

Bcl-2 is a direct target of $m i R-16$. Previous studies have indicated that miRNAs regulate gene expression by decreasing mRNA translation, increasing mRNA degradation, or both $(13,14)$. To examine if miR-16 regulated $\mathrm{Bcl}-2$ expression, RT-qPCR and western blot analyses were performed to investigate the expression of $\mathrm{Bcl}-2$ following miR-16 transfection.
The miR-16 mimic decreased Bcl-2 protein expression in a dose-dependent manner (Fig. 2A). By contrast, a miR-16 inhibitor increased Bcl-2 protein expression (Fig. 2B).

miR-16 inhibits Bcl-2 expression in hypoxia/SD-induced BM-MSC apoptosis. To confirm whether elevated miR-16 expression decreased Bcl-2 expression in hypoxia/SD-induced BM-MSCs apoptosis, miR-16 mimics were transfected. As a control, BM-MSCs were transfected with a miR-16 inhibitor or were placed in hypoxic/SD conditions with no transfection. The miR-16 mimic increased miR-16 expression in a dose-dependent manner; no significant difference in Bcl-2 mRNA expression was detected (Fig. 2C). Western blot analysis confirmed that $\mathrm{Bcl}-2$ protein expression was significantly decreased in miR-16 mimic-transfected cells, compared with control cells (Fig. 2D). Notably, the decreased expression of miR-16 in BM-MSCs resulted in increased expression of $\mathrm{Bcl}-2$ protein. Taken together, these results demonstrate that increased miR-16 expression results in decreased Bcl-2 protein expression.

Overexpression of miR-16 reduces cell viability and inhibits cell proliferation. To examine the potential role of miR-16 in regulating cell viability and proliferation, MTT and EdU assays were performed. Notably, it was determined that the number of EdU-positive cells (green) in the miR-16 mimic group were reduced compared with the inhibitor group (Fig. 3A and B). Additionally, cell viability significantly increased in the 

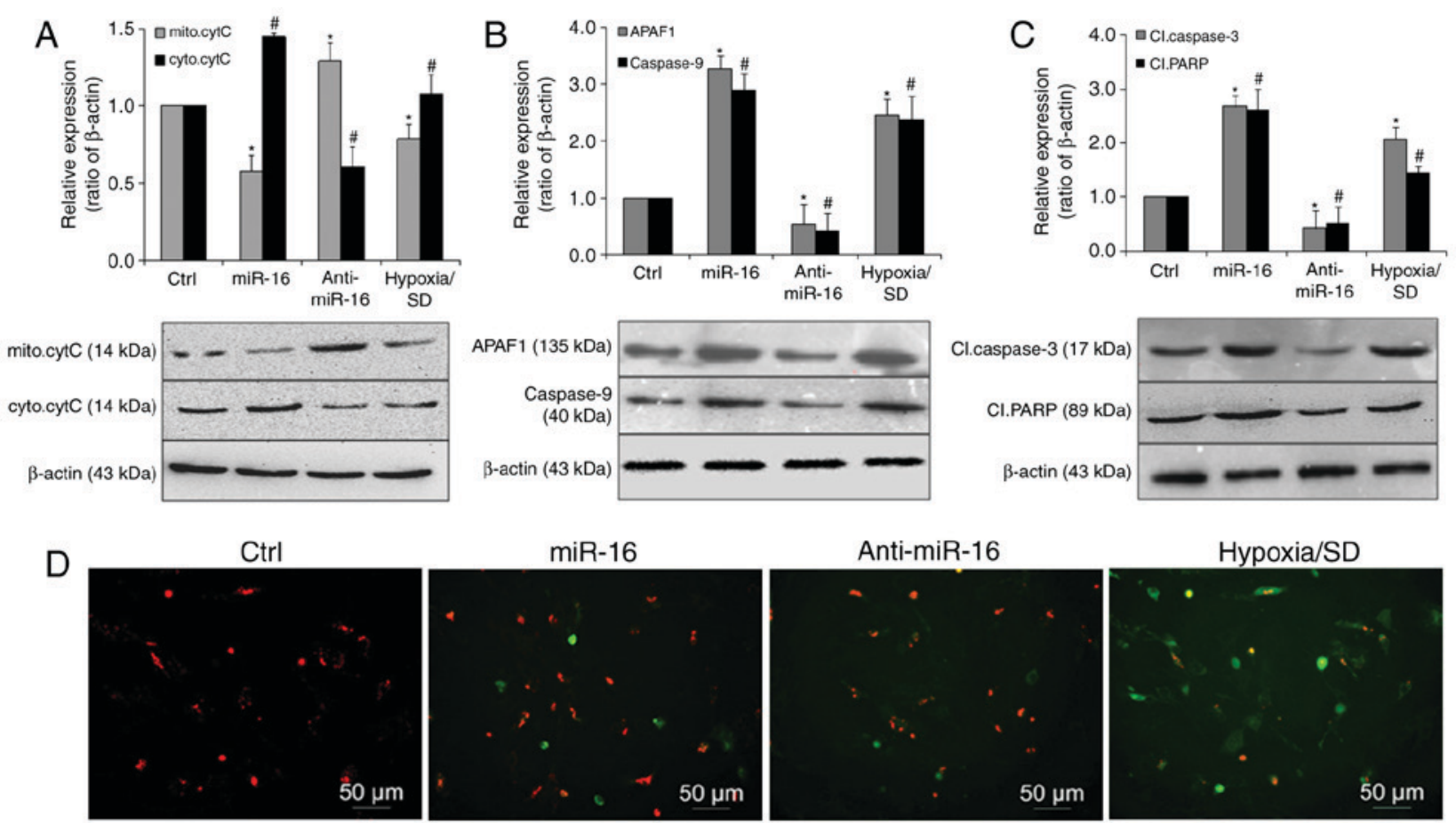

Figure 4. miR-16 promoted apoptosis via activation of a mitochondrial pathway involving APAF-1/caspase-9/PARP. (A) Representative immunoblots and quantitative analysis of mitochondrial or cytosolic cytochrome $\mathrm{c}$ levels in BM-MSCs transfected with miR-16 mimics or inhibitor. The expression of (B) APAF-1 and caspase-9, and (C) cleaved caspase-3 and cleaved PARP was significantly increased in cells transfected with miR-16 mimics. (D) BM-MSCs (red) transfected with miR-16 mimics or inhibitor were then exposed to hypoxia/SD for $24 \mathrm{~h}$, and mitochondrial membrane (green) potential was assessed using a JC-1 MMP Assay. Magnification, x100. Data are presented as the mean \pm standard deviation of three independent experiments. "P<0.05 and ${ }^{\#} \mathrm{P}<0.05$ vs. the respective Ctrl group. miR-16, microRNA-16, APAF-1, apoptotic protease activating factor-1; PARP, poly (ADP ribose) polymerase; BM-MSC, bone marrow-derived mesenchymal stem cell; SD, serum deprivation; cytC, cytochrome c; mito, mitochondrial; cyto, cytoplasmic; Cl, cleaved; MMP, mitochondrial membrane potential.

A

$$
\text { A }
$$
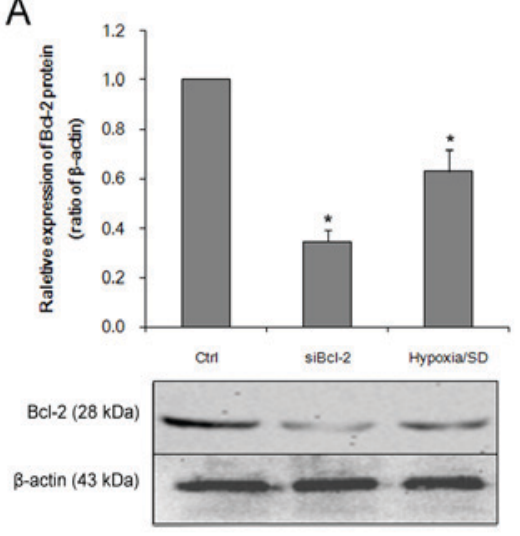

$\mathrm{D}$

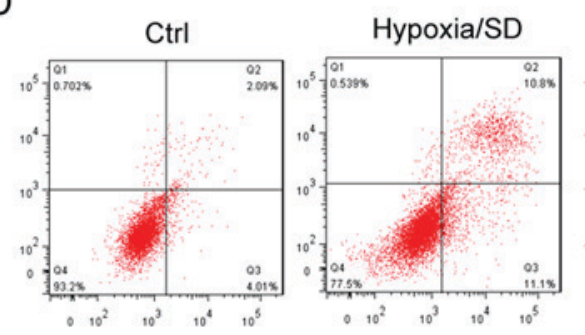

B

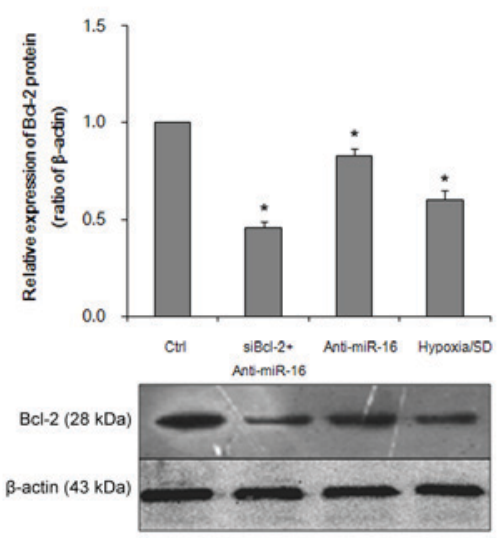

C
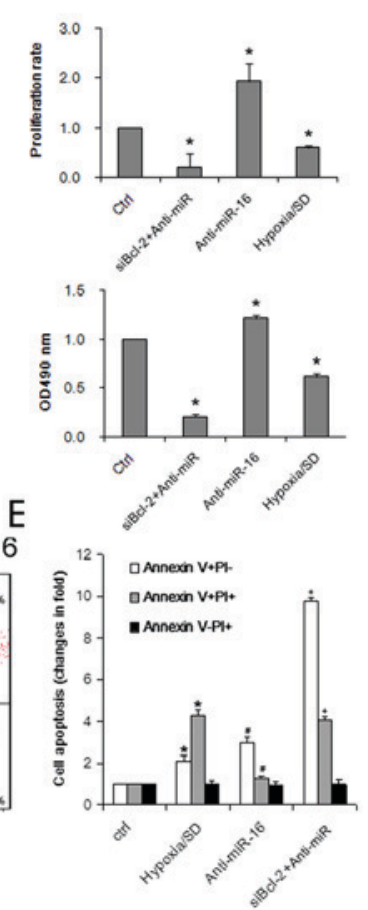

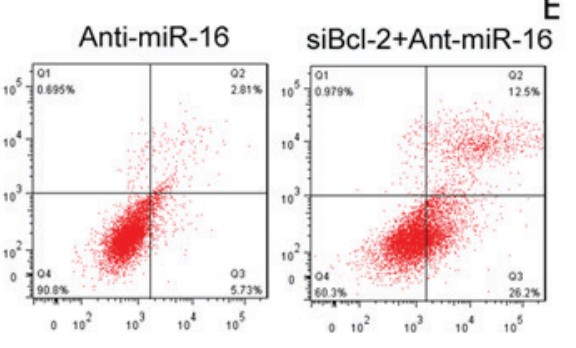

Figure 5. Bcl-2 was involved in miR-16-induced apoptosis under hypoxic/SD conditions. (A) Silencing of Bcl-2 expression was confirmed by western blot analysis in BM-MSCs transfected with siBcl-2 or (B) co-transfected with siBcl-2 and miR-16 inhibitor. (C) BM-MSC proliferation and viability following transfection of miR-16 inhibitor and siBcl-2 was determined by EdU and MTT assays. (D) Flow cytometry analysis of apoptosis following miR-16 inhibitor and siBcl-2 transfection. (E) Fold change in cell apoptosis. Data are presented as the mean \pm standard deviation of three independent experiments. $\mathrm{P}<0.05$ vs. ctrl, ${ }^{\#} \mathrm{P}<0.05$ vs. Hypoxia/SD, ${ }^{+} \mathrm{P}<0.01$ vs. anti-miR-16. Bcl-2, B-cell lymphoma 2; miR-16, microRNA-16; BM-MSC, bone marrow-derived mesenchymal stem cell; siBcl-2, Bcl-2 small interfering RNA; SD, serum deprivation; Ctrl, control. 
presence of the miR-16 inhibitor, compared with the miR-16 mimic or hypoxia/SD (Fig. 3C).

Overexpression of miR-16 promotes the apoptosis of BM-MSCs. To examine the role of miR-16 in apoptosis promotion, miR-16 expression was increased or decreased in BM-MSCs by transfecting cells with a miR-16 mimic, or a miR-16 inhibitor for $72 \mathrm{~h}$, respectively. After $24 \mathrm{~h}$ of incubation under hypoxic/SD conditions, miR-16 led to a marked pro-apoptotic effect. Flow cytometry also demonstrated that there was a significant decrease in the percentage of apoptotic cells in the anti-miR-16 group (Fig. 3D and E). Exposure of BM-MSCs to hypoxia/SD resulted in a $7.88 \pm 0.46$-fold increase in apoptotic rate when compared with controls, whereas $40.1 \pm 0.32 \%$ of cells transfected with miR-16 were apoptotic. In contrast, only $10.9 \pm 0.23 \%$ of cells transfected with anti-miR-16 were apoptotic (Fig. 3E).

miR-16 exerts it pro-apoptotic effects via activation of the mitochondrial pathway of apoptosis. Cytochrome $\mathrm{c}$ is a key mediator of apoptosis that is released from mitochondria, and its release is inhibited by the presence of $\mathrm{Bcl}-2$ incorporated into the outer membrane of organelles $(28,29)$. Therefore, to investigate if the intrinsic apoptotic pathway was activated by miR-16, cell lysates fractions were subjected to western blot analysis. The results revealed that cytoplasmic cytochrome c was significantly increased in the miR-16 mimic transfected group. By contrast, its expression was markedly decreased in the miR-16 inhibitor transfected group, compared with the controls (Fig. 4A). Caspase-9 and APAF-1 demonstrated a similar expression pattern, with significantly increased levels observed in the miR-16 mimic transfected group (Fig. 4B) Furthermore, cleaved caspase-3 and cleaved PARP were also significantly upregulated in the miR-16 mimic group and reduced in the miR-16 inhibitor group, compared with the control (Fig. 4C). The effect of miR-16 was further ascertained by examining the MMP (Fig. 4D). Compared with the control group, cells transfected with the miR-16 mimic displayed noticeable alterations in MMP. These results suggest that miR-16 may be involved in the apoptotic process of BM-MSCs induced by hypoxia/SD through activation of the mitochondrial pathway of apoptosis, via APAF-1/caspase-9/PARP.

Bcl-2 knockdown promotes apoptosis and suppresses proliferation. To explore miR-16-mediated regulation of $\mathrm{Bcl}-2$ in BM-MSCs apoptosis and growth, Bcl-2 siRNA was used to knockdown Bcl-2 expression. As presented in Fig. 5A, treatment with Bcl-2 siRNA significantly reduced the expression of Bcl-2 protein. To determine if dysregulation of Bcl-2 expression was involved in the regulation of miR-16-induced cell apoptosis, BM-MSCs were co-transfected with a miR-16 inhibitor and Bcl-2 siRNA. The effect of Bcl-2 siRNA on Bcl-2 expression was confirmed by western blot analysis (Fig. 5B). MTT and EdU assays revealed that Bcl-2 siRNA inhibited proliferation (Fig. 5C) and cell viability (Fig. 5D) to an extent that resembled the inhibitory effects of miR-16 overexpression on BM-MSCs (Fig. 3). It was demonstrated that the apoptosis-promoting effects of miR-16 were partially elevated by Bcl-2 siRNA and Bcl-2 siRNA mimicked the effects of miR-16 (Fig. 5E) suggesting that Bcl-2 may be involved in miR-16-induced apoptosis under conditions of hypoxia/SD.

\section{Discussion}

The results of the present study revealed that miR-16 expression was significantly upregulated under hypoxic/SD conditions in BM-MSCs. Downregulation of miR-16 resulted in decreased apoptosis and enhanced cell proliferation. Bcl-2 was identified as a direct and functional target of miR-16. Further experiments indicated that miR-16 induced an intrinsic apoptosis pathway via APAF-1/caspase-9/PARP. The present study may lead to the discovery of a more optimized and effective target in MSC-based therapy for myocardial infarction. BM-MSC transplantation is a potential therapeutic approach to improve cardiac function in patients following myocardial infarction (4-6). However, the repair and regeneration of cardiomyocytes and the restoration of heart function are limited by the poor survival of engrafted BM-MSCs in the infarcted area. In the present study, it was revealed that under conditions of hypoxia/SD, designed to mimic the in vivo conditions occurring in ischemic myocardium, apoptosis of BM-MSC was triggered, as detected by phosphatidylserine translocation to the cell surface and loss of membrane integrity. This was demonstrated by Annexin V/PI stain, positive Annexin V staining demonstrated phosphatidylserine translocation to the cell surface and positive PI staining indicated loss of membrane integrity. This finding was confirmed by flow cytometry analysis. The results of the present study were in agreement with the results obtained by Zhu et al (26), who reported that transplanted BM-MSCs may be lost due to apoptosis induced by hypoxia/SD. In addition to producing ATP, mitochondria are also key regulators of apoptosis in a variety of cell types (29). The results of the current study indicated that the release of cytochrome $\mathrm{c}$ from mitochondria was involved in subsequent caspase activation during apoptosis.

Recent evidence has revealed significant roles of miRNAs, including miR-16, in numerous forms of cardiovascular disease (31). In miRNA-induced apoptosis, expected targets include genes that encode proteins with apoptotic activity or targets that act as negative regulators of cell proliferation and survival $(16,32)$. miR-16 has been demonstrated to be involved in apoptosis in other cell types (16-18) and to inhibit various key regulators of cell cycle progression $(33,34)$. For example, miR-16 contributes to BM-MSC differentiation into myocardial cells in a cardiac niche by enhancing G1 phase arrest (35). Accumulating data have presented miR-16 as an appealing target for further investigation $(19,20)$. In the present study, the effect of miR-16 on BM-MSC apoptosis and proliferation under conditions of hypoxia/SD was focused on. Previous studies have demonstrated that miR-16 negatively regulates Bcl-2 expression via imperfect complementarity with Bcl-2 mRNA, and, therefore promotes apoptosis (19,36). Bcl-2 blocks the mitochondrial release of cytochrome $\mathrm{c}$ and inhibits the activation of caspase- 9 by the cytoplasmic scaffolding protein APAF-1 (18). In the current study, Bcl-2 knockdown with siRNA mimicked the effects of miR-16 overexpression in suppressing cell proliferation and inducing cell apoptosis. These data indicate the specific role of Bcl-2 in miR-16 regulation of apoptosis. miR-16 has been reported to target a number of genes (18-20). In future studies, it is planned to identify if other apoptotic regulatory genes are involved in miR-16-induced apoptosis. 
To elucidate the association between the upregulation of miR-16 expression under hypoxic/SD conditions and downregulation of its target Bcl-2 protein, RT-qPCR and western blot analysis was performed in the present study. Overexpression of miR-16 led to decreased Bcl-2 protein expression and increased apoptosis. In BM-MSCs transfected with miR-16 mimics, increased apoptosis, release of cytochrome c, and cleavage of pro-caspase-9 and PARP was observed, indicating that the reduction in $\mathrm{Bcl}-2$ protein expression by miR-16 was sufficient to initiate the apoptotic process. These results suggest that miR-16 induced apoptosis in BM-MSCs through activation of the APAF-1/caspase-9/PARP pathway.

In conclusion, the present study indicated that exposure to hypoxic/SD conditions significantly increased the expression of miR-16 in BM-MSCs.miR-16 overexpression may promote apoptosis through downregulation of the anti-apoptotic $\mathrm{Bcl}-2$ protein. Additionally, it was demonstrated that miR-16 suppressed the expression of Bcl-2 at the post-transcriptional level and induced apoptosis by activating the intrinsic APAF-1/caspase-9/PARP pathway. Furthermore, inhibition of miR-16 provided protection to BM-MSCs against hypoxia/SD-induced apoptosis. Therefore, miR-16 may be a potential therapeutic target for supporting cellular transplantation therapy in myocardial infarction.

\section{Acknowledgements}

The authors would like to thank Dr Wei Liu (The Key Laboratory of Myocardial Ischemia Mechanism and Treatment, Harbin Medical University, Ministry of Education, Harbin, Heilongjiang, China) for her excellent technical assistance and helpful discussions.

\section{Funding}

The present study was supported by the National Natural Science Foundation of China (grant no. 3001-30400432).

\section{Availability of data and materials}

The datasets used and/or analyzed during the current study are available from the corresponding author on reasonable request.

\section{Authors' contributions}

JR contributed to the experimental design, performed the molecular biology experiments and the statistical analysis, and drafted the manuscript. SF participated in the design of the study and performed the statistical analysis. YW revised the manuscript and performed some of the experiments. BL was responsible for MSC transfection and statistical analysis. BY participated in the design of the study. SL conceived the study and participated in its design and coordination, and helped to draft the manuscript. All authors read and approved the final manuscript.

\section{Ethics approval and consent to participate}

All experimental animal procedures were approved by the Ethical Committee on Animal Care and Use of Harbin Medical University (Harbin, China).

\section{Consent for publication}

Not applicable.

\section{Competing interests}

The authors declare that they have no competing interests.

\section{References}

1. Pittenger MF, Mackay AM, Beck SC, Jaiswal RK, Douglas R, Mosca JD, Moorman MA, Simonetti DW, Craig S and Marshak DR: Multilineage potential of adult human mesenchymal stem cells. Science 284: 143-147, 1999.

2. Small EM and Olson EN: Pervasive roles of microRNAs in cardiovascular biology. Nature 469: 336-342, 2011.

3. Afzal MR, Samanta A, Shah ZI, Jeevanantham V, Abdel-Latif A, Zuba-Surma EK and Dawn B: Adult bone marrow cell therapy for ischemic heart disease: Evidence and insights from randomized controlled trials. Circ Res 117: 558-575, 2015.

4. Strauer BE and Steinhoff G: 10 years of intracoronary and intramyocardial bone marrow stem cell therapy of the heart: from the methodological origin to clinical practice. J Am Coll Cardiol 58: 1095-1104, 2011.

5. Li W, Ma N, Ong LL, Nesselmann C, Klopsch C, Ladilov Y, Furlani D, Piechaczek C, Moebius JM Lützow K, et al: Bcl-2 Engineered MSCs Inhibited Apoptosis and Improved Heart Function. Stem Cells 25: 2118-2127, 2007.

6. Stamm C, Westphal B, Kleine HD, Petzsch M, Kittner C, Klinge $\mathrm{H}$, Schümichen $\mathrm{C}$, Nienaber $\mathrm{CA}$, Freund $\mathrm{M}$ and Steinhoff G: Autologousbone-marrow stem-cell transplantation for myocardial regeneration. Lancet 361: 45-46, 2003.

7. Menasché $P$, Hagège AA, Vilquin JT, Desnos M, Abergel E, Pouzet B, Bel A, Sarateanu S, Scorsin M, Schwartz K, et al: Autologous skeletal myoblast transplantation for severe postinfarction left ventricular dysfunction. J Am Coll Cardiol 41: 1078-1083, 2003

8. Leistner DM, Fischer-Rasokat U, Honold J, Seeger FH, Schächinger V, Lehmann R, Martin H, Burck I, Urbich C, Dimmeler $\mathrm{S}$, et al: Transplantation of progenitor cells and regeneration enhancement in acute myocardial infarction (TOPCARE-AMI): Final 5-year results suggest long-term safety and efficacy. Clin Res Cardiol 100: 925-934, 2011.

9. Toma C, Pittenger MF, Cahill KS, Byrne BJ and Kessler PD: Human mesenchymal stem cells differentiate to a cardiomyocyte phenotype in the adult murine heart. Circulation 105: 93-98, 2002.

10. Hare JM, Traverse JH, Henry TD, Dib N, Strumpf RK, Schulman SP, Gerstenblith G, DeMaria AN, Denktas AE, Gammon RS, et al: A randomized, double-blind, placebo-controlled, dose-escalation study of intravenous adult human mesenchymal stem cells (prochymal) after acute myocardial infarction. J Am Coll Cardiol 54: 2277-2286, 2009

11. Bartel DP: MicroRNAs: Genomics, Biogenesis, Mechanism, and Function. Cell 116: 281-297, 2004.

12. Carè A, Catalucci D, Felicetti F, Bonci D, Addario A, Gallo P, Bang ML, Segnalini P, Gu Y, Dalton ND, et al: MicroRNA-133 controls cardiac hypertrophy. Nat Med 13: 613-618, 2007.

13. Valencia-Sanchez MA, Liu J, Hannon GJ and Parker R: Control of translation and mRNA degradation by miRNAs and siRNAs. Genes Dev 20: 515-524, 2006.

14. Davis JA, Saunders SJ, Mann M and Backofen R: Combinatorial microRNA target predictions. Nat Genet 37: 495-500, 2005

15. RI, Calin GA and Croce CM: miR-15a and miR-16-1 in cancer: discovery, function and future perspectives. Cell Death Differ 17: 215-220, 2010.

16. van Rooij E, Marshall WS and Olson EN: Toward MicroRNA-based therapeutics for heart disease: The sense in antisense. Circ Res 103: 919-928, 2008.

17. Bandi N, Zbinden S, Gugger M, Arnold M, Kocher V, Hasan L, Kappeler A, Brunner T and Vassella E: miR-15a and miR-16 are implicated in cell cycle regulation in a Rb-dependent manner and are frequently deleted or down-regulated in non-small cell lung cancer. Cancer Res 69: 5553-5559, 2009.

18. Cimmino A, Calin GA, Fabbri M, Iorio MV, Ferracin M, Shimizu M, Wojcik SE, Aqeilan RI, Zupo S, Dono M, et al: miR-15 and miR-16 induce apoptosis by targeting BCL2. Proc Natl Acad Sci USA 102: 13944-13949, 2005. 
19. Guo CJ, Pan Q, Li DG, Sun H and Liu BW: miR-15b and miR-16 are implicated in activation of the rat hepatic stellate cell: An essential role for apoptosis. J Hepatol 50: 766-778, 2009.

20. Xia L, Zhang D, Du R, Pan Y, Zhao L, Sun S, Hong L, Liu J and Fan D: miR-15b and miR-16 modulate multidrug resistance by targeting BCL2 in human gastric cancer cells. Int J Cancer 123: 372-379, 2008

21. Czabotar PE, Lessene G, Strasser A and Adams JM: Control of apoptosis by the BCL-2 protein family: Implications for physiology and therapy. Nat Rev Mol Cell Biol 15: 49-63, 2014.

22. Bonci D, Coppola V, Musumeci M, Addario A, Giuffrida R Memeo L, D'Urso L, Pagliuca A, Biffoni M, Labbaye C, et al: The miR-15a-miR-16-1 cluster controls prostate cancer by targeting multiple oncogenic activities. Nat Med 14: 1271-1277, 2008.

23. Yang J, Liu X, Bhalla K, Kim CN, Ibrado AM, Cai J, Peng TI, Jones DP and Wang X: Prevention of Apoptosis by Bcl-2: Release of cytochrome c from mitochondria blocked. Science 275 1129-1132, 1997

24. Kluck RM, Bossy-Wetzel E, Green DR and Newmeyer DD: The release ofcytochrome $\mathrm{c}$ from mitochondria: A primary site for Bcl-2 regulation of apoptosis. Science 275: 1132-1136, 1997.

25. Li P, Nijhawan D, Budihardjo I, Srinivasula SM, Ahmad M, Alnemri ES and Wang X: Cytochrome c and dATP-dependent formation of apaf-1/caspase- 9 complex initiates an apoptotic protease cascade. Cell 91: 479-489, 1997.

26. Zhu W, Chen J, Cong X, Hu S and Chen X: Hypoxia and serum deprivation-induced apoptosis in mesenchymal stem cells. Stem Cells 24: 416-425, 2006.

27. Liu J, Wang Y, Du W, Liu W, Liu F, Zhang L, Zhang M, Hou M, Liu K, Zhang S and Yu B: Wntl inhibits hydrogen peroxide-induced apoptosis in mouse cardiac stem cells. PLoS One 8: e58883, 2013.

28. Livak KJ and Schmittgen TD. Analysis of relative gene expression data using real time quantitative PCR and the 2(-Delta Delta C(t)) method. Methods 25: 402-408, 2001.
29. Green DR and Reed JC: Mitochondria and apoptosis. Science 281: 1309-1311, 1998.

30. Liu WH, Liu JJ, Wu J, Zhang LL, Liu F, Yin L, Zhang MM and $\mathrm{Yu}$ B: Novel mechanism of inhibition of dendritic cells maturation by mesenchymal stem cells via interleukin -10 and the JAK1/STAT3 Signaling Pathway. PLoS One 8: e55487, 2013.

31. Han D, Huang W, Li X, Gao L, Su T, Li X, Ma S, Liu T, Li C, Chen J, et al: Melatonin facilitates adipose-derived mesenchymal stem cells to repair the murine infarcted heart via the SIRT1 signaling pathway. J Pineal Res 60: 178-192, 2016.

32. Barwari T, Joshi A and Mayr M: MicroRNAs in cardiovascular disease. J Am Coll Cardiol 68: 2577-2584, 2016.

33. Yan X, Liang H, Deng T, Zhu K, Zhang S, Wang N, Jiang X, Wang X, Liu R, Zen K, et al: The identification of novel targets of miR-16 and characterization of their biological functions in cancer cells. Mol Cancer 12: 92, 2013.

34. Roccaro AM, Sacco A, Thompson B, Leleu X, Azab AK, Azab F, Runnels J, Jia X, Ngo HT, Melhem MR, et al: MicroRNAs $15 \mathrm{a}$ and 16 regulate tumor proliferation in multiple myeloma. Blood 113: 6669-6680, 2009.

35. Liu JL, Jiang L, Lin QX, Deng CY, Mai LP, Zhu JN, Li XH, Yu XY, Lin SG and Shan ZX: MicroRNA 16 enhances differentiation of human bone marrow mesenchymal stem cells in a cardiac niche toward myogenic phenotypes in vitro. Life Sci 90 : 1020-1026, 2012.

36. Calin GA, Dumitru CD, Shimizu M, Bichi R, Zupo S, Noch E, Aldler H, Rattan S, Keating M, Rai K, et al: Frequent deletions and down-regulation of micro-RNA genes miR15 and miR16 at 13q14 in chronic lymphocytic leukemia. Proc Natl Acad Sci USA 99: 15524-15529, 2002.

This work is licensed under a Creative Commons Attribution-NonCommercial-NoDerivatives 4.0 International (CC BY-NC-ND 4.0) License. 